\title{
Pain Research - Methods and Protocols - Second Edition
}

\author{
Z. David Luo (Editor). Springer. Humana Press. ISBN 978-1-61779-560-2
}

\author{
Bradley Kerr, PhD
}

Received: 20 September 2012/ Accepted: 1 October 2012/Published online: 18 October 2012

(C) Canadian Anesthesiologists' Society 2012

This second edition of Pain Research - Methods and Protocols is an excellent compendium of chapters outlining the most current techniques used to study the neurobiology of pain. This new version edited by Dr. David Luo includes chapters that outline more advanced techniques for cellular recording, genomic analysis, and other molecular approaches that were unavailable when the first edition was compiled. In addition, the book includes a number of excellent chapters that describe the rationale and methodology for new animal models addressing trigeminal neuralgia and orofacial pain, cancer pain, pain related to direct spinal cord trauma (avulsion and contusion injuries), as well as novel peripheral nerve and chronic inflammatory pain conditions.

The text is organized logically into sections. The first several chapters deal with novel molecular and electrophysiological approaches, and subsequent chapters deal with various injury models and are arranged into sections that move from headache and orofacial pain into spinal cord injuries, peripheral nerve injuries, inflammatory/ arthritis models, and bone cancer pain. Each chapter includes a brief introduction to the rationale and experimental context for the development or refinement of each model and then provides a step-by-step outline of the materials and methods required to carry out each model. Additionally, illustrations and example diagrams are used effectively by highlighting key technical points and outcomes in each of the various models.

In the book's preface, Dr. Luo describes the rapid advancement of experimental approaches and medical technology that are available to bench scientists in general but are being applied to pain research more specifically. The significance of these advancements lies mainly in the acceleration of research findings thus "allowing the integrated investigations of pain processing mechanisms at the single cell and/or molecular level". However, pain does not stop at the single-cell level but requires a multidisciplinary approach to tackle the complex behavioural responses to injury and to assess the novel analgesic compounds adequately. Thus, there is the need for well-characterized animal models. It is in this regard that some of the basic approaches used in the laboratory are expertly brought together concurrently with highlighting the more recent developments in modelling very distinct pain syndromes.

Pain Research - Methods and Protocols is aimed not only at the novice researcher with little experience in the field but also at bringing new techniques and approaches to the pain research community as a whole. The clear organizational structure of the text makes this a highly achievable goal.

Competing interests None declared.

B. Kerr, $\mathrm{PhD}(\square)$

University of Alberta, Edmonton, AB, Canada

e-mail: bradley.kerr@ualberta.ca 